\title{
RANDOM WALKS WHOSE CONCAVE MAJORANTS OFTEN HAVE FEW FACES
}

\author{
ZHIHUA QIAO and J. MICHAEL STEELE
}

\begin{abstract}
We construct a continuous distribution $G$ such that the number of faces in the smallest concave majorant of the random walk with $G$-distributed summands will take on each natural number infinitely often with probability one. This investigation is motivated by the fact that the number of faces $F_{n}$ of the concave majorant of the random walk at time $n$ has the same distribution as the number of records $R_{n}$ in the sequence of summands up to time $n$. Since $R_{n}$ is almost surely asymptotic to $\log n$, the construction shows that despite the equality of all of the one-dimensional marginals, the almost sure behaviors of the sequences $\left\{R_{n}\right\}$ and $\left\{F_{n}\right\}$ may be radically different.
\end{abstract}

\section{INTRODUCTION}

If $X_{i}, i=1,2, \ldots$ is a sequence of independent random variables with a continuous distribution $G$, then the number of records

$$
R_{n}=\max \left\{k: X_{i_{1}}<X_{i_{2}}<\ldots<X_{i_{k}}, 1 \leq i_{1}<i_{2}<\cdots<i_{k} \leq n\right\}
$$

was studied in Rényi (1962) and was found to have the same distribution as

$$
\xi_{1}+\xi_{2}+\cdots+\xi_{n}
$$

where $\left\{\xi_{i}: i=1,2, \ldots\right\}$ is a sequence of independent Bernoulli random variable that satisfy $P\left(\xi_{k}=1\right)=1 / k$. Goldie (1989) later observed that as a consequence of Spitzer's combinatorial lemma as generalized by Brunk (1964) that the number of faces of the concave majorant of the random walk $S_{k}=X_{1}+X_{2}+\cdots+X_{k}$, $1 \leq k \leq n$, also has the same distribution as $R_{n}$; that is, if one lets $F_{n}$ denote the number of pieces in the smallest piecewise linear concave majorant of the set of points $\mathcal{S}_{n}=\left\{(0,0),\left(1, S_{1}\right), \ldots,\left(n, S_{n}\right)\right\}$, then one has $P\left(R_{n} \leq t\right)=P\left(F_{n} \leq t\right)$ for all $t \in \mathbb{R}$ and all integers $1 \leq n<\infty$.

By a standard Borel-Cantelli argument, one finds from the Bernoulli sum representation (1) of $R_{n}$ and the monotonicity $R_{n} \leq R_{n+1}$ that

$$
\lim _{n \rightarrow \infty} R_{n} / \log n=1 \quad \text { with probability } 1,
$$

so from Goldie's observation that $R_{n} \stackrel{d}{=} F_{n}$ for each $\mathrm{n}$, one might expect an analogous strong law for the sequence $\left\{F_{n}: n=1,2, \ldots\right\}$, despite the fact that the sequence $\left\{F_{n}: n=1,2, \ldots\right\}$ is not monotone. In Steele (2002) it was suggested the

Date: April 7, 2005.

1991 Mathematics Subject Classification. Primary: 60D05; Secondary: 60C05,68U05,62G99.

Key words and phrases. Spitzer's combinatorial lemma, random walk, convex hull,convex minorant, concave majorant. 


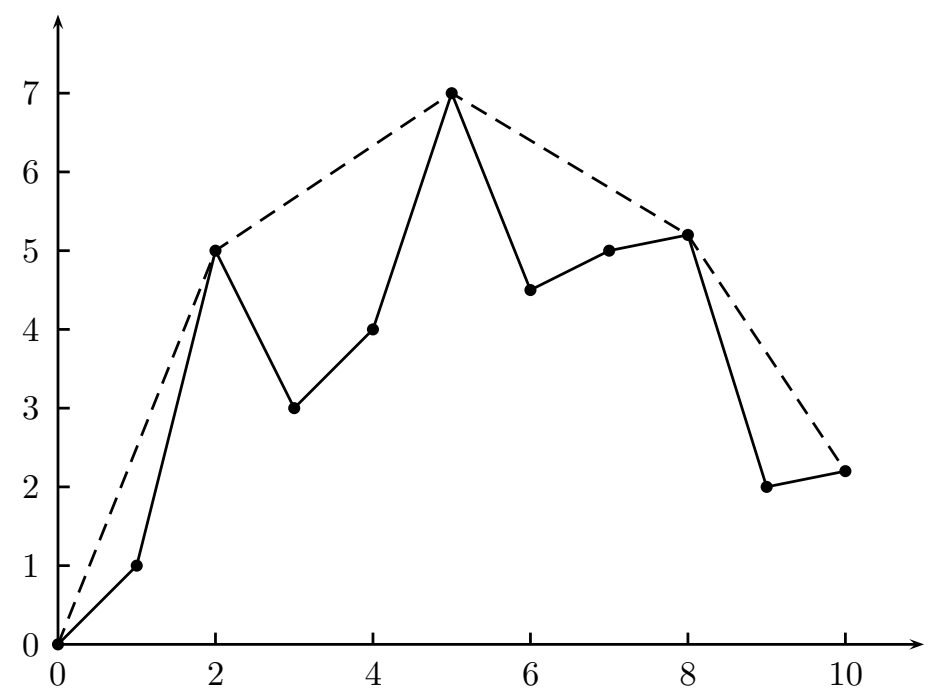

FiguRE 1. The concave majorant of a random walk

limit law for records (2) might not extend to the face process $\left\{F_{n}: n=1,2, \ldots\right\}$, and the main goal of this note is to confirm a particularly strong version of this conjecture.

Theorem 1. There exists a continuous distribution function $G$ such that if the random variables $X_{i}, i=1,2, \ldots$ are independent with $P\left(X_{i} \leq x\right)=G(x)$ for all $i=1,2, \ldots$ and if $F_{n}$ denotes the number of faces of the least concave majorant of the first $n$ steps of the random walk $S_{k}, 1 \leq k \leq n$, then

$$
P\left(F_{n}=m \quad \text { i.o. }\right)=1 \quad \text { for each value of } m=1,2, \ldots .
$$

In particular, the concave majorant of the set $\mathcal{S}_{n} \subset \mathbb{R}^{2}$ will be a single line infinitely often with probability one.

The behavior described by the relation (3) contrasts about as sharply with the limit (2) as one could imagine, despite the fact that the marginal distributions of $F_{n}$ and $R_{n}$ are equal for each $n$.

\section{Two Constructive Lemmas}

The basic idea is that one can construct a continuous distribution $G$ such that infinitely often the summand $X_{i}$ is so large that it completely overwhelms the cumulative contributions of all of the preceding summands. The implementation of this idea rests on two simple technical lemmas. To begin, we take an arbitrary sequence of integers $2 \leq n_{1}<n_{2}<\cdots$, and consider independent discrete random variables $Y_{i}, i=1,2, \ldots$ such that $P\left(Y_{i}=n_{k}\right)=p_{k}$ for all $i \geq 1$ and $k \geq 1$. Our technical lemmas tell us about events that are unlikely to take place in conjunction with the first time that an element of the sequence $\left\{Y_{i}\right\}$ is equal to $n_{k}$. 
Lemma 1. For $\alpha>1$, let $c_{\alpha}$ be chosen such that $p_{k}=c_{\alpha}(k !)^{-\alpha}$ is a probability measure on $\{1,2, \ldots\}$. If one sets

$$
B_{k, t}=\left\{Y_{t}=n_{k}\right\} \cap\left\{Y_{s} \neq n_{k} \text { for all } 1 \leq s<t\right\} \cap\left\{\max _{s<t} Y_{s}>n_{k}\right\},
$$

then for $A_{k}=\bigcup_{t=1}^{\infty} B_{k, t}$ one has $P\left(A_{k} \quad\right.$ i.o. $)=0$.

Proof. If we set $\alpha_{k}=\sum_{j=1}^{k-1} p_{j} /\left(1-p_{k}\right)$, then by independence of the $\left\{Y_{k}\right\}$ we have

$$
P\left(B_{k, t}\right)=p_{k}\left(1-p_{k}\right)^{t-1}\left(1-\alpha_{k}^{t-1}\right) .
$$

Since the events $\left\{B_{k, t}\right\}_{t=1}^{\infty}$ are disjoint, we also have

$$
\begin{aligned}
\sum_{k=1}^{\infty} P\left(A_{k}\right) & =\sum_{k=1}^{\infty} \sum_{t=1}^{\infty} P\left(B_{k, t}\right)=\sum_{k=1}^{\infty} p_{k}\left(\frac{1}{p_{k}}-\frac{1}{1-\left(1-p_{k}\right) \alpha_{k}}\right) \\
& =\sum_{k=1}^{\infty} \frac{1-\sum_{j=1}^{k} p_{j}}{1-\sum_{j=1}^{k-1} p_{j}}=\sum_{k=1}^{\infty} \frac{\sum_{j=k+1}^{\infty} p_{j}}{\sum_{j=k}^{\infty} p_{j}} \\
& \leq \sum_{k=1}^{\infty} \frac{1}{(k+1)^{\alpha}}<\infty,
\end{aligned}
$$

and the proof is completed by applying the Borel-Cantelli Lemma.

REMARK: One should note that with the choice $p_{k}=c_{\alpha}(k !)^{-\alpha}$ the condition $\alpha>1$ cannot be dropped. For example, one can easily check that if $p_{k}=e / k !$, then the sum of the $P\left(A_{k}\right)$ diverges. On the other hand, a referee has observed that an interesting alternative that does work here (and in the next lemma) is given by $p_{k}=q^{k^{2}}$ for any $0<q<1$. With this choice the inequalities (4) and (5) are unchanged but the last line is somewhat simplified. Specifically, with $p_{k}=q^{k^{2}}$ the bound in (6) can be replaced by $q^{3}+q^{5}+q^{7}+\cdots<\infty$.

Lemma 2. Suppose the sequence $\left\{n_{k}\right\}$ satisfies the gap condition $n_{k} / n_{k-1}>k / p_{k}$ for all $k \geq 2$. If we have

$$
E_{k, t}=\left\{Y_{t}=n_{k}\right\} \cap\left\{Y_{s}<n_{k}, \text { for all } 1 \leq s<t\right\} \quad \text { and } \quad E_{k}=\cup_{t \geq n_{k} / n_{k-1}} E_{k, t},
$$

then we have $P\left(E_{k}\right.$ i.o. $)=0$.

Proof. We have $P\left(E_{k, t}\right)=p_{k}\left(\sum_{i=1}^{k-1} p_{i}\right)^{t-1}$, so by disjointness we find

$$
\begin{aligned}
\sum_{k=1}^{\infty} P\left(E_{k}\right) & =\sum_{k=1}^{\infty} \sum_{t=\left\lceil\frac{n_{k}}{n_{k-1}}\right\rceil}^{\infty} p_{k}\left(\sum_{i=1}^{k-1} p_{i}\right)^{t-1}=\sum_{k=1}^{\infty} p_{k} \frac{\left(\sum_{i=1}^{k-1} p_{i}\right)^{\left\lceil\frac{n_{k}}{n_{k-1}}\right\rceil-1}}{1-\sum_{i=1}^{k-1} p_{i}} \\
& \leq \sum_{k=1}^{\infty} p_{k} \frac{\left(1-p_{k}\right)^{\left\lceil\frac{n_{k}}{n_{k-1}}\right\rceil-1}}{p_{k}} \leq \sum_{k=1}^{\infty}\left(1-p_{k}\right)^{\left(k / p_{k}\right)-1} .
\end{aligned}
$$

From the bound $1-x \leq e^{-x}$ and the geometric sum, one sees the last sum is not larger than $\left(1-e^{-1}\right)^{-1}$, so the Borel-Cantelli Lemma again completes the proof. 


\section{Proof of Theorem 1}

We now say that a random time $\tau$ is good provided that

- $\tau \geq 2$,

- $Y_{j}<Y_{\tau}$ for all $1 \leq j<\tau$, and

- for $n_{k}$ such that $Y_{\tau}=n_{k}$ one has $\tau<n_{k} / n_{k-1}$,

so, the main point of Lemmas 1 and 2 is that they immediately imply that with probability one there exists an infinite sequence $\tau_{1}<\tau_{2}<\ldots$ of good times. To complete the proof of Theorem 1 we just need to connect the existence of these good times to the geometry of the concave majorant of an appropriate random walk.

Specifically, we let $U_{n}, n=1,2, \ldots$ denote a sequence of independent random variables with the uniform distribution on $(0,1)$, and we set $X_{n}=Y_{n}+U_{n}$, where the $Y_{n}$ are as before. We will now focus on the random walk $S_{n}=X_{1}+X_{2}+\cdots+X_{n}$ and confirm that the continuous distribution $G(x)=P\left(X_{k} \leq x\right)$ satisfies the claims of Theorem 1 .

The key geometric fact of the construction, fits snugly into a single line:

$$
\text { if } \tau \text { is a good time, then } F_{\tau}=1 \text {. }
$$

To see why this assertion is true, we first note that

$$
\frac{S_{\tau}}{\tau}>\frac{n_{k}+(\tau-1) n_{1}}{\tau}>n_{k-1}+n_{1}-n_{1} / \tau \geq n_{k-1}+1,
$$

where in the last step we use the facts that $n_{1} \geq 2$ and $\tau \geq n_{1}$. We then note that for all $t<\tau$ we have

$$
\frac{S_{t}}{t}<\frac{t\left(n_{k-1}+1\right)}{t}=n_{k-1}+1
$$

and the truth of the assertion (7) follows immediately from the bounds (8) and (9).

By assertion (7) and the almost sure existence of an infinite sequence of good times, we therefore find that $F_{n}=1$ infinitely often with probability one. Now we only need to check that for each $m \geq 1$ we also have $F_{n}=m$ infinitely often with probability one. The basic idea here is that we get infinitely many independent tries at an event that has probability that is uniformly bounded away from zero.

More formally, since the summands $\left\{X_{k}=Y_{k}+U_{k}: k=1,2, \ldots\right\}$ are nonnegative, elementary geometry tells us that for each good time $\tau$ and for each $m \geq 2$ that the event

$$
S_{\tau} / \tau>X_{\tau+1}>X_{\tau+2}>\cdots>X_{\tau+m-1}
$$

implies the event $F_{\tau+m-1}=m$. Also, the event $X_{\tau+1}>X_{\tau+2}>\cdots>X_{\tau+m-1}$ has probability greater than that of the event

$$
C_{m}=\left\{Y_{\tau+1}=Y_{\tau+2}=\cdots=Y_{\tau+m-1}=n_{1}\right\} \cap\left\{U_{\tau+1}>U_{\tau+2}>\cdots>U_{\tau+m-1}\right\},
$$

and $C_{m}$ has probability $p_{1}^{m-1} /(m-1) !=\delta_{m}>0$, which does not depend on $\tau$. Moreover, by (8) one always has $S_{\tau} / \tau>n_{1}+1$ provided that $Y_{\tau} \geq n_{2}$, so along the infinite sequence of good times $\tau_{j}, j=2,3, \ldots$ one can find has infinitely many opportunities of observing $F_{\tau+m-1}=m$ that are independent and that have probability $\delta_{m}>0$. Thus, by the law of large numbers one finds that with probability one, we have $F_{n}=m$ for infinitely many $n$, and the proof of Theorem 1 is complete. 


\section{A Final Observation}

The distribution $G$ constructed here suffices to show that one cannot expect regular behavior of $F_{n}$ at the same level of generality that one finds regularity for $R_{n}$. Nevertheless, the sequence $F_{n}$ may not always be badly behaved. Under nice conditions - say, for example, when the summands are exponentially distributed - one may be able to prove a useful limit law for $F_{n}$.

In the hunt for such a law, it may be useful to note that for summands with a continuous distribution one always has

$$
\limsup _{n \rightarrow \infty} \frac{F_{n}}{\log n} \geq 1 \quad \text { with probability one. }
$$

Moreover, from the equality of the distributions of $R_{n}$ and $F_{n}$ and the representation (1) for $R_{n}$, one has the useful large deviation bound:

$$
P\left(\left|F_{n}-H_{n}\right| \geq \epsilon H_{n}\right) \leq 2 \exp \left(-\epsilon^{2} H_{n} / 4\right) \quad \text { for all } \quad 0 \leq \epsilon \leq 1 / 2,
$$

where $H_{n}=1+1 / 2+1 / 3+\cdots+1 / n$. In fact, the bound (11) follows immediately from the usual concentration inequalities for Bernoulli sums, say, for example, Bennet's inequality (Bennett (1962), equation (8b)). Finally, from (11) one easily proves (10) with the Borel-Cantelli lemma and a subsequence argument.

\section{REFERENCES}

G. Bennett, Probability inequalities for sums of independent random variables, $J$. Amer. Statist. Soc., 57 (1962), 33-45.

H.D. Brunk, A Generalization of Spitzer's Combinatorial Lemma, Z. Wahrscheinlichkeitstheorie, 2 (1964), 395-405.

C.M. Goldie, Records, permutations and greatest convex minorants, Math. Proc. Camb. Phil. Soc., 106 (1989), 169-177.

A. Rényi, On the extreme elements of observations, MTA III Oszt. Közl, 12 (1962), 105-121. (also, available in Selected Papers of Alfréd Rényi, Vol. 3, 50-66, P. Turán, editor, Akademiai Kaidó, Budapest, 1976.)

F. Spitzer, A combinatorial lemma and its application to probability theory, Trans. Amer. Math. Soc., 82 (1956), 323-339

J.M. Steele, The Bohnenblust-Spitzer Algorithm and Its Applications, Journal of Computational and Applied Mathematics, 142 (2002), 235-249.

Department of Statistics, Wharton School, University of Pennsylvania, Steinberg Hall-Dietrich Hall 3000, University of Pennsylvania, Philadelphia PA 19104

E-mail address: steele@wharton.upenn.edu, zhqiao@wharton.upenn.edu 